\title{
Ensayos
}

Pensar en Movimiento:

Revista de Ciencias del Ejercicio y la Salud ISSN 1659-4436

Vol. 16, No.2, pp. 1- 13

Abre $1^{\circ}$ de julio, cierra 31 de diciembre, 2018

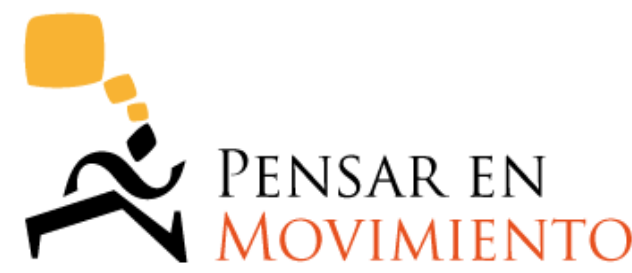

REVISTA DE CIENCIAS DEL EJERCICIO Y LA SALUD

\section{ADAPTACIÓN DEL MODELO DE PLANIFICACIÓN ATR AL ENTORNO FORMATIVO. APLICACIÓN EN CLASES DE TAEKWONDO}

\section{ADAPTATION OF THE ATR PLANNING MODEL TO THE TRAINING ENVIRONMENT. IMPLEMENTATION IN TAEKWONDO CLASSES}

\section{ADAPTAÇÃO DO MODELO DE PLANIFICAÇÃO ATR PARA O MEIO FORMATIVO. APLICAÇÃO NAS AULAS DE TAEKWONDO}

\author{
Pedro Carazo-Vargas \\ pedro.carazo@ucr.ac.cr \\ Centro de Investigación en Ciencias del Movimiento Humano y Escuela de Educación \\ Física y Deportes, Universidad de Costa Rica, Costa Rica \\ Envío original: 2018-03-09; reenviado: 2018-06-27; \\ aceptado: 2018-07-31; publicado: 2018-09-21.
}

Doi: http://dx.doi.org/10.15517/pensarmov.v16i2.32689

\section{RESUMEN}

Carazo-Vargas, P. (2018). Adaptación del modelo de planificación ATR al entorno formativo. Aplicación en clases de taekwondo. Pensar en Movimiento: Revista de Ciencias del Ejercicio y la Salud, 16(2), 1-13. La planificación ATR es un método de organización del entrenamiento deportivo que se aplica en busca de conseguir la óptima forma competitiva en atletas de distintas disciplinas. La presente disertación expone las principales características de este modelo de planificación y describe una posible manera de aplicarlo en el entorno educativo y de formación base. Se ejemplifica el empleo de dicha adaptación en clases de taekwondo, entorno en el cual ha sido utilizado exitosamente. La verificación empírica relacionada con la eficacia de la adaptación de este modelo es necesaria.

Palabras clave: recreación física; formación deportiva; deporte base; artes marciales. 


\section{ABSTRACT}

Carazo-Vargas, P. (2018). Adaptation of the ATR planning model to the training environment. Implementation in taekwondo clases. PEnSAR en Movimiento: Revista de Ciencias del Ejercicio y la Salud, 16(2), 1-13. The Accumulation, Transmutation, Realization (ATR) Planning model is a sports training periodization method applied to achieve optimal physical shape prior to competitions in athletes of different disciplines. The main characteristics of this planning model are described here, as well as a possible way to apply the model in the educational and basic training environments. Its implementation in taekwondo training is used as an example, which has proven to be successful. The effectiveness of implementing this model must be empirically verified.

Keywords: physical recreation; sports training; grassroots sport; martial arts.

\section{RESUMO}

Carazo-Vargas, P. (2018). Adaptação do modelo de planificação ATR para o meio formativo. Aplicação nas aulas de taekwondo. Pensar en Movimiento: Revista de Ciencias del Ejercicio y la Salud, 16(2), 1-13. A planificação ATR é um método de organização de treinamento esportivo que se aplica em busca de atingir a melhor forma competitiva em atletas de distintas disciplinas. A presente dissertação expõe as principais características deste modelo de planificação e descreve uma possível maneira de aplicálo no meio educativo e de formação base. É exemplificado o emprego desta adaptação nas aulas de taekwondo, meio no qual tem sido utilizado com muito sucesso. A verificação empírica relacionada com a eficácia da adaptação deste modelo é necessária.

Palavras-chave: recreação física; formação esportiva; esporte base; artes marciais.

En el ámbito competitivo de alto nivel, se pretende conseguir el mejor resultado posible en los eventos en que intervienen los deportistas. En procura de esta meta, se estructura el entrenamiento de manera que exista interconexión entre los estímulos para generar las adaptaciones que conduzcan a mejorar el rendimiento deportivo (GonzálezRavé, Pablos-Abella y Navarro-Valdivieso, 2014).

La periodización deportiva, concebida como un sinónimo de planear y analizar el entrenamiento, basa sus orientaciones en la experiencia reportada por prominentes figuras de la Unión Soviética de la década de 1960; posteriormente, ha continuado evolucionando con el aporte de muchos científicos y entrenadores, siempre con el objetivo de maximizar el rendimiento atlético (Issurin, 2010).

No obstante, una mayor cantidad de personas practican deporte sin un objetivo de rendimiento deportivo. Aunque el deporte genera importantes beneficios en sus practicantes, el entrenamiento y la competición incrementan el riesgo de sufrir lesiones que pueden producir un impacto negativo en a nivel físico y psicosocial (Houston, Hoch y Hoch, 2016). La exigencia y el riesgo que conlleva la preparación deportiva con fines 
competitivos hacen que el deporte practicado por rendimiento no sea adecuado para todas las personas. En el conjunto de personas que realizan deporte sin un objetivo de rendimiento deportivo, es posible identificar a las que lo hacen con fines de recreación y salud, o bien, como formación base para competir en una posterior etapa.

Los deportes, juegos y actividades físicas corresponden a uno de los tipos de actividad recreativa utilizada por las personas para aprovechar su tiempo libre, pasar un tiempo agradable y recibir beneficios en su desarrollo integral y bienestar general (Salazar, 2007). Además de buscar un positivo aprovechamiento del tiempo libre, el objetivo de obtener un beneficio en la salud es otra fuente de motivación que impulsa a muchas personas a realizar deporte. Existe suficiente evidencia que respalda cómo la práctica regular de actividad física y ejercicio conlleva numerosos beneficios físicos y mentales, generando un impacto positivo en aspectos tales como la mortalidad, el riesgo de desarrollar enfermedades cardiovasculares, prevenir la diabetes y algunos tipos de cáncer, la alta presión arterial y desordenes psicológicos (Garber et al., 2011).

El deporte base es una etapa de gran relevancia para el desarrollo de un competidor, ya que precisamente aprovecha que la persona se encuentra en formación para impulsar correctamente el dominio de los gestos técnicos y el fortalecimiento físico; paralelamente, también se refuerza la diversión y el placer por la práctica de actividad física (García et al., 2018). No obstante, si bien la actividad deportiva ha demostrado su potencial para contribuir en múltiples dominios de funcionamiento, incluyendo el físico, el psicológico, el cognitivo, el académico y el social, sus beneficios no son inherentes a su práctica. Aunque no se encuentre en el ámbito del alto rendimiento, dependiendo del contexto en el cual se desarrolle, también puede generar violencia, competitividad excesiva, estrés, lesiones y agotamiento (Theokas, 2009). Debido a esta situación, el entorno en la cual se desarrolla la práctica deportiva es de gran relevancia.

La adaptación del modelo de planificación de bloques que a continuación se describe se considera de gran utilidad, ya que puede contribuir en la reducción del riesgo de generar efectos negativos al practicar deporte y facilitar la consecución de los objetivos anteriormente descritos para las personas que desean realizar actividad física con fines de recreación y salud, o bien, los propósitos perseguidos por los jóvenes que se inician en la práctica deportiva con fines competitivos.

Para conseguir describir la adaptación del modelo de periodización ATR (Acumulación; Transformación; Realización) en el entorno formativo, inicialmente se describen las principales características de la planificación deportiva y, específicamente, de esta metodología de organización del entrenamiento. Posteriormente, se presenta la manera en la cual se pueden organizar los mesociclos, los microciclos y las sesiones de práctica al perseguir fines formativos o recreativos; finalmente, se presentan ejemplos prácticos del empleo de esta metodología en clases de taekwondo.

\section{DESARROLLO}

La periodización pretende la división de toda la temporada en unidades de entrenamiento llamadas macrociclos, mesociclos microciclos y sesiones (ordenadas de 
mayor a menor duración), de modo que se presente un ordenamiento adecuado de los ejercicios, su especificidad, volumen, intensidad y frecuencia de entrenamiento, con el objetivo de alcanzar la mejor forma deportiva previa a la intervención en las competencias (Bompa y Buzzichelli, 2015).

De acuerdo con los planteamientos teóricos del entrenamiento deportivo, se presentan dos maneras principales en las cuales evoluciona la carga: los modelos lineales y los no lineales (González-Ravé et al., 2014; Ranisavljevic e llic, 2010). El modelo lineal se ha asociado con el sistema de planificación tradicional y los modelos no lineales con los sistemas de planificación contemporáneos; no obstante, no se puede ignorar que en el modelo tradicional también se presentan fluctuaciones en las cargas de trabajo (Issurin, 2010).

La planificación tradicional del entrenamiento tiene a Matveev como su principal referente. Este educador planteó el modelo clásico de periodización fundamentado en su “Teoría General del Entrenamiento Deportivo", la cual se sustenta en las leyes biológicas y, en especial, en el síndrome de adaptación al estrés (González-Ravé et al., 2014). El objetivo que persigue la planificación tradicional es alcanzar la forma deportiva óptima durante las competencias; para ello, la periodización se basa en tres etapas consecutivas:

a. Construcción: desarrollo de las capacidades del deportista.

b. Mantenimiento: sostenimiento de dichas capacidades de forma interactiva.

c. Pérdida de la forma deportiva: facilitación para iniciar un nuevo ciclo.

El cumplimiento de estos postulados se manifiesta con el desarrollo de tres periodos: a) el periodo preparatorio, que busca la adquisición de la forma deportiva; b) el periodo competitivo, que pretende el mantenimiento de la forma deportiva; y c) el periodo de transición, que tiene la finalidad de facilitar la recuperación del organismo para comenzar otro ciclo de forma deportiva (Campos y Cervera, 2011).

En la actualidad se han presentado diversos cambios en la estructura de la competición internacional, tales como la profesionalización de los atletas, el aumento de la cantidad de competiciones anuales, el entorno económico y social que se desprende del deporte de rendimiento y el desarrollo del conocimiento y, con ello, la posibilidad de participación de un equipo de atención multidisciplinaria (González-Ravé et al., 2014).

Ante este entorno, varios investigadores en el área del entrenamiento deportivo (Carazo-Vargas, González-Ravé, Moncada-Jiménez y Newton, 2015; González-Ravé et al., 2014; Issurin, 2010) han especulado sobre diversas debilidades en la periodización tradicional, entre las que señalan:

a. Requiere una reducción considerable en el volumen de entrenamiento.

b. Existe discrepancia respecto al beneficio del entrenamiento simultáneo de múltiples habilidades.

c. Los atletas altamente calificados podrían recibir un estímulo insuficiente al ser entrenados bajo este sistema.

d. Se imposibilita mantener frecuentes picos de forma durante la temporada.

-4 -

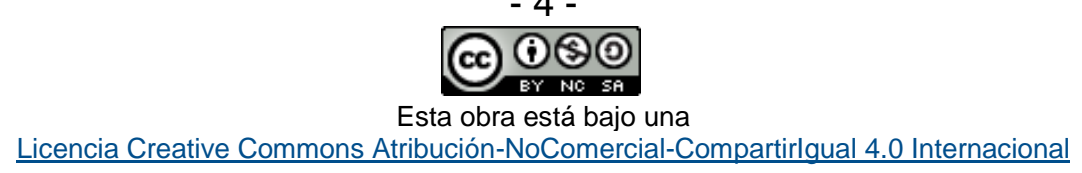


El modelo ATR, también llamado de cargas concentradas, es un método de periodización contemporáneo que intenta responder a las demandas del deporte actual. Organiza el entrenamiento en tres tipos de mesociclos, en los cuales se aplican variaciones constantes en las cargas de trabajo para desarrollar una reducida cantidad de objetivos en cada bloque. Los tres tipos especializados de mesociclos son: a) Acumulación b) Transformación y c) Realización. Dependiendo de su objetivo, la duración de los mesociclos fluctúa entre las dos y las seis semanas (Issurin, 2010).

El mesociclo de acumulación pretende desarrollar capacidades físicas y contribuir en el aprendizaje, perfeccionamiento y corrección de la técnica deportiva. Se caracteriza por la aplicación de importantes cargas de trabajo de tipo específico, que usualmente tienden a aplicarse con un alto volumen y moderada intensidad (Carazo-Vargas et al., 2015; González-Ravé et al., 2014).

El mesociclo de transformación tiene el objetivo de adaptar las capacidades y conocimientos específicos adquiridos en el mesociclo de acumulación a las necesidades propias de la competición; por ejemplo, en el taekwondo el entrenamiento de la potencia y la táctica toman el lugar que tenían la fuerza y la técnica en el anterior mesociclo. Se suelen utilizar ejercicios específicos, adaptados a las características que se van a requerir en la competición (Carazo-Vargas et al., 2015; González-Ravé et al., 2014).

El mesociclo de realización busca dirigir los esfuerzos a obtener el máximo rendimiento durante la competición. Se trabaja con gran intensidad y mediano volumen, aplicando los ejercicios según las características que se presentarán durante la competición (Carazo-Vargas et al., 2015; González-Ravé et al., 2014).

Los microciclos son estructuras más pequeñas, que se prolongan entre cuatro y siete días, y son de gran importancia, pues su estructura y contenido guía la consecución de los objetivos de entrenamiento perseguidos por los atletas (Bompa y Buzzichelli, 2015). En la Tabla 1 se presenta la descripción de las principales características de los diferentes tipos de microciclos que componen los mesociclos de un macrociclo, según el modelo de preparación ATR.

Las sesiones son las unidades básicas de entrenamiento. Según la magnitud de la carga y el tiempo necesario para recuperarse, se pueden categorizar del 1 al 5 . Las sesiones con carga 1 pretenden recuperación y en menos de 12 horas ya se ha presentado una regeneración completa luego del esfuerzo. Las sesiones con carga 2 buscan el mantenimiento de las adaptaciones conseguidas y la recuperación completa se da entre las 12 y las 24 horas una vez finalizado el entrenamiento. Las sesiones con cargas 3, 4 y 5 pretenden el desarrollo de adaptaciones, de acuerdo con el esfuerzo realizado, la recuperación se presentará luego de las 24 horas y, en el caso de las sesiones con carga 5, se puede prolongar por más de tres días (González-Ravé et al., 2014).

La adaptación de este modelo de periodización al entorno formativo contempla como eje primordial facilitar un espacio para que la persona disfrute y aprenda mediante la ejecución de la actividad; asimismo, tomando en cuenta que la persona que decida continuar entrenando con fines competitivos debe contar con un adecuado desarrollo 
técnico-táctico, pretende promover el desarrollo y corrección en la ejecución de las destrezas.

Tabla 1

Características microciclos de entrenamiento

\begin{tabular}{|c|c|c|c|c|}
\hline Microciclo & Objetivo & Carga trabajo & $\begin{array}{l}\text { Duración } \\
\text { (días) }\end{array}$ & $\begin{array}{l}\text { Sesiones } \\
\text { características }\end{array}$ \\
\hline Ajuste & $\begin{array}{l}\text { Preparar al deportista } \\
\text { para el siguiente } \\
\text { mesociclo }\end{array}$ & $\begin{array}{l}\text { Medio, importante. } \\
\text { Disminuye } \\
\text { intensidad }\end{array}$ & $4-7$ & $\begin{array}{l}\text { Mantenimiento } \\
\text { y desarrollo }\end{array}$ \\
\hline Carga & $\begin{array}{l}\text { Crear adaptaciones } \\
\text { Cargas trabajo altas }\end{array}$ & Importante, grande & 7 & Desarrollo \\
\hline Impacto & $\begin{array}{l}\text { Estimular las } \\
\text { adaptaciones al máximo }\end{array}$ & Grande, extremo & 7 & Desarrollo \\
\hline Activación & $\begin{array}{l}\text { Preparar para la } \\
\text { competencia Recuperar }\end{array}$ & $\begin{array}{l}\text { Bajo, medio } \\
\text { Volumen bajo } \\
\text { Intensidad alta }\end{array}$ & $3-7$ & $\begin{array}{l}\text { Mantenimiento } \\
\text { y recuperación }\end{array}$ \\
\hline Competitivo & $\begin{array}{l}\text { Organizar la } \\
\text { competencia }\end{array}$ & $\begin{array}{l}\text { Depende de } \\
\text { competición }\end{array}$ & $3-9$ & $\begin{array}{l}\text { Mantenimiento } \\
\text { y recuperación }\end{array}$ \\
\hline Recuperación & $\begin{array}{l}\text { Crear condiciones para } \\
\text { recuperación }\end{array}$ & $\begin{array}{l}\text { Bajo. Volumen e } \\
\text { intensidad baja }\end{array}$ & $3-7$ & $\begin{array}{l}\text { Mantenimiento } \\
\text { y recuperación }\end{array}$ \\
\hline
\end{tabular}

Fuente: González-Ravé et al. (2014).

Se considera que el modelo puede ser aprovechado tanto por personas que realizan la actividad con fines de salud y esparcimiento como por aquellas que podrían manifestar un interés por la competición. En las etapas iniciales, las prácticas deben ir dirigidas a contribuir a desarrollar adecuados gestos técnicos y a fomentar el disfrute por la actividad, ya que ambos elementos pueden representar el abandono de la práctica deportiva si no han recibido la adecuada atención. La propuesta tiene el potencial de contribuir en ambos aspectos.

A diferencia del modelo tradicional ATR, en el que la adecuada manipulación del volumen, la duración, la frecuencia, la intensidad y densidad de las cargas físicas de entrenamiento son los elementos condicionantes del logro de los objetivos perseguidos, la presente adaptación prioriza en la demanda cognitiva requerida para realizar las actividades que se distribuyen en las distintas sesiones de entrenamiento.

Paralelamente y en forma dependiente de las características e intereses del grupo de personas a la cuales esté dirigida la actividad, se buscará un mejoramiento de las cualidades físicas; no obstante, el perfeccionamiento técnico-táctico siempre será prioritario. Considerando que la mayor parte de personas que practican deporte sin objetivos de rendimiento lo hará con un menor volumen total de práctica que quienes sí lo hacen, se considera inconveniente planificar sesiones exclusivas de desarrollo físico, 
pues además de que podrían ser insuficientes para lograr adaptaciones importantes, estarían en detrimento del desarrollo técnico-táctico.

Los mesociclos mantienen su nombre y duración. El mesociclo de acumulación pretende mejorar el repertorio técnico de la persona; el entrenamiento buscará el perfeccionamiento de las destrezas ya conocidas y la incorporación de nuevas destrezas y situaciones a las ya dominadas.

Es importante tener en cuenta que para lograr el perfeccionamiento técnico-táctico y mantener la motivación de los practicantes, se deben tener en cuenta las diferencias individuales. Los gestos practicados deben representar un desafío para la persona, pero esta también debe ser capaz de percibir un progreso al realizarlos. Así, se deben tener en cuenta estos factores, ya que no es recomendable acumular altas cargas de repeticiones cuando un gesto técnico aún no se ha aprendido correctamente o para el cual no se está preparado, pues podrían generar grandes vicios de ejecución. El trabajo individualizado o en subgrupos de personas con similar nivel son opciones para responder a esta necesidad.

Durante el mesociclo de transformación se mantiene el objetivo de adaptar las capacidades y conocimientos específicos adquiridos en el mesociclo de acumulación a las necesidades propias de la competición; no obstante, las cualidades físicas siempre tendrán un aspecto secundario. El entrenamiento físico se realizará paralelamente a la aplicación de técnicas y estrategias ejecutadas en situaciones de competencia. Debe considerarse que la contigüidad de los bloques de acumulación con transformación no asegura la continuidad del proceso; se cumplirán si los contenidos internos de la planificación son realmente progresivos en un contexto metodológico.

El mesociclo de realización busca la ejecución de las destrezas con una máxima eficiencia en el contexto real de competencia. La adaptación del modelo mantiene la particularidad de perseguir múltiples puestas a punta durante el año, elemento que se considera una fuente de motivación para las personas practicantes.

Es importante tener la perspectiva de que tanto la preparación deportiva con fines formativos como competitivos corresponden a procesos a largo plazo. La progresión del individuo se dará de acuerdo con la manera en la cual se vaya manifestando su potencial; no se debería acelerar el progreso de una persona sin que esté preparada para ello. Si bien en el mesociclo de realización el trabajo se podría concentrar en perfeccionar los gestos más eficientes, al comenzar un nuevo macrociclo se continuarán practicando tanto las opciones más efectivas como las menos eficientes para ese momento dado.

Debido a las características de la población a la que se prepara, el mesociclo de realización no necesariamente coincidirá con una competición; no obstante, esto no representa un inconveniente, se pretende que el mesociclo de realización esté asociado con la consecución de una meta de aprendizaje, ejecución previamente establecida, o bien, con la realización de una presentación a un público meta definido.

Al completarse un macrociclo (los tres tipos de mesociclos) se dará continuidad al proceso, depurando las destrezas que aún no se han logrado ejecutar satisfactoriamente en situación de competencia o incorporando nuevas destrezas. En la Tabla 2 se presenta la descripción de las principales características de los diferentes tipos de microciclos de la 
adaptación del modelo ATR al entorno formativo. El objetivo del microciclo se direcciona hacia las características de las actividades técnico-tácticas programadas y la carga de las sesiones corresponde a una valoración de la exigencia de las mismas realizada por la persona responsable de la programación.

Tabla 2

Características microciclos modelo adaptado

\begin{tabular}{llc}
\hline Microciclo & Objetivo & Carga subjetiva \\
\hline Ajuste & $\begin{array}{l}\text { Repaso, nada nuevo, nada técnico que represente } \\
\text { esfuerzo }\end{array}$ & $1-2$ \\
Carga & $\begin{array}{l}\text { Introducción de elementos nuevos o variantes } \\
\text { Impacto }\end{array}$ & $3-4$ \\
Activación & $\begin{array}{l}\text { Sesiones complejas, aspectos técnicos con esfuerzo con alta intensidad física y técnica sin } \\
\text { introducir esfuerzo técnico }\end{array}$ & $4-5$ \\
Competitivo & Ejecución al máximo rendimiento & 2 \\
Recuperación & $\begin{array}{l}\text { Sesiones recreativas, juegos o actividades sin } \\
\text { relación directa con el deporte }\end{array}$ & 1 \\
\hline
\end{tabular}

Fuente: elaboración propia.

Aunque no se dispone de evidencia que respalde un óptimo ordenamiento de los distintos tipos de microciclos, se respetan los principios originales de modelo colocando los microciclos de ajuste al inicio de los mesociclos y programando una mayor cantidad de microciclos de carga, los cuales preceden a los de impacto. Los microciclos de activación se ubican antes de los competitivos (que son exclusivos de los mesociclos de realización), mientras que los de recuperación se pueden programar luego de un mesociclo de competición o al iniciar un nuevo macrociclo.

La adaptación del modelo de periodización descrito nace de la experiencia, de más 15 años, en programar y dirigir lecciones de taekwondo para grupos de niños mayores de seis años, adolescentes y personas adultas mayores. Se carece de sustento para recomendar el modelo con niños menores a los seis años. A continuación, se describen algunos ejemplos de su aplicación.

Una de las destrezas más importantes en la práctica del taekwondo y otras artes marciales son las "formas" (i.e., rutinas preestablecidas de técnicas de ataque y contraataque ejecutadas hacia oponentes imaginarios); para su enseñanza, bajo el presente modelo, en un mesociclo de acumulación se buscaría el aprendizaje independiente de cada una de las destrezas que componen la forma (e.g., defensa arriba, defensa abajo, patada de frente, etc.), en el mesociclo de transformación se integrarían las técnicas aprendidas en la secuencia de movimientos requeridas y en el mesociclo de realización se esperaría la ejecución de la "forma" completa de la mejor manera posible.

Dando continuidad al ejemplo anteriormente mencionado, en un sucesivo macrociclo se le puede dar seguimiento al perfeccionamiento de la misma "forma" o bien 
se podría seguir el mismo patrón en cada uno de los mesociclos, pero enfatizando en elementos más específicos que no se lograron en el anterior ciclo (e.g., precisión de movimientos, energía, velocidad, etc.). En la Tabla 3 se presentan ejemplos de posibles actividades a realizar en el entrenamiento de las "formas" en los microciclos de ajuste, carga e impacto para un mesociclo de acumulación y otro de transformación.

Tabla 3

Ejemplo actividades para el entrenamiento de las "formas" según microciclo

\begin{tabular}{|c|c|c|}
\hline \multirow{2}{*}{ Microciclo } & \multicolumn{2}{|c|}{ Mesociclo } \\
\hline & Acumulación & Transformación \\
\hline Ajuste & $\begin{array}{l}\text { Práctica de destrezas conocidas } \\
\text { (e.g., puño al medio) }\end{array}$ & Práctica de defensas y posiciones \\
\hline Carga & $\begin{array}{l}\text { Enseñanza de defensas (e.g., } \\
\text { arriba, abajo) y posiciones (e.g., } \\
\text { corta, larga) de manera } \\
\text { independiente }\end{array}$ & $\begin{array}{l}\text { Enseñanza de secuencia de la } \\
\text { forma en la cual se integran } \\
\text { defensas y posiciones }\end{array}$ \\
\hline Impacto & $\begin{array}{l}\text { Ejecución simultánea de defensas } \\
\text { y posiciones }\end{array}$ & $\begin{array}{l}\text { Ejecución simultánea de la } \\
\text { secuencia aprendida frente a otra } \\
\text { persona (acciones opuestas entre } \\
\text { personas) }\end{array}$ \\
\hline
\end{tabular}

Fuente: elaboración propia.

En la Tabla 4 se ejemplifican posibles actividades a ejecutar en distintos microciclos que pretenden el desarrollo de contenidos concernientes a la defensa personal.

La aplicación del modelo con técnicas de combate deportivo seguirá los mismos principios descritos que con las "formas y la defensa personal.

Si bien por facilidad descriptiva, los ejemplos de las actividades que se presentaron podrían corresponder a estrategias de enseñanza relacionadas con la reproducción, es relevante señalar que las metodologías de enseñanza basadas en la producción también son aplicables y que las mismas podrían generar beneficios adicionales.

En el mesociclo de acumulación se pretenderá que las personas perfeccionen el gesto técnico de las acciones ya dominadas e incorporen nuevas combinaciones o variantes. Al respecto, es importante destacar que el fin último es la aplicación efectiva de las acciones en competencia. Si la persona es capaz de efectuar satisfactoriamente la destreza en situación de entrenamiento (e.g., pateando un saco) pero no en competición, la técnica no se puede catalogar como dominada.

En el mesociclo de transformación las prácticas se concentran en las acciones técnicas con mayor dominio y su aplicación en diferentes escenarios (e.g., distancias de combate respecto al oponente, lugar de ubicación en el área de competencia, diferencias en la puntuación de competencia, etc.). En el mesociclo de realización se busca que la 
ejecución de las acciones practicadas se efectúe en el contexto de competición, de modo que la persona esté preparada para participar en ella. Ya sea que se dé la intervención en una competencia oficial o que se realice una simulación en el mismo ambiente de entrenamiento, la evaluación del alcance de las metas perseguidas en el macrociclo es esencial para definir los objetivos de trabajo del siguiente ciclo.

Tabla 4

Ejemplo actividades para el entrenamiento de defensa personal según microciclo

\begin{tabular}{|c|c|c|}
\hline \multirow[t]{2}{*}{ Microciclo } & \multicolumn{2}{|c|}{ Mesociclo } \\
\hline & Acumulación & Transformación \\
\hline Ajuste & $\begin{array}{l}\text { Práctica contra agarre a muñeca. } \\
\text { Contrario agarra con mano } \\
\text { contraria (técnica ya aprendida) }\end{array}$ & - \\
\hline Carga & $\begin{array}{l}\text { Enseñanza contra agarre a } \\
\text { muñeca. Contrario agarra con } \\
\text { mano del mismo lado. }\end{array}$ & $\begin{array}{l}\text { Oponente agarra muñeca con } \\
\text { cualquiera de las dos manos y se } \\
\text { debe tomar la decisión de la técnica } \\
\text { a aplicar. No hay parejas, personas } \\
\text { se desplazan libremente y cualquier } \\
\text { persona toma iniciativa de realizar el } \\
\text { agarre. }\end{array}$ \\
\hline Impacto & $\begin{array}{l}\text { Contrario agarra muñeca con } \\
\text { cualquiera de las dos manos y se } \\
\text { debe tomar la decisión de la } \\
\text { técnica a aplicar. }\end{array}$ & $\begin{array}{l}\text { Similar al ejercicio anterior, aunque } \\
\text { siempre se enfatizará en el agarre } \\
\text { de muñecas, habrá libertad para } \\
\text { otras acciones de ataque que ya se } \\
\text { hubieran practicado en anteriores } \\
\text { macrociclos (e.g., agarre al cuello). }\end{array}$ \\
\hline
\end{tabular}

Fuente: elaboración propia.

Los microciclos de carga son los más abundantes durante el proceso de preparación; en el mesociclo de acumulación se pueden enfocar en el perfeccionamiento técnico de nuevas destrezas, o bien, dejar que las acciones técnicas permanezcan en un rol secundario y aprovecharlas para buscar mejoras en la condición física.

Los microciclos de impacto estarán presentes únicamente en mesociclos de acumulación y transformación. Las sesiones desarrolladas durante estas semanas pueden ser diseñadas en contextos distintos a los presentes en competición (e.g., contraataques a varios oponentes, estímulos auditivos, series de 20 repeticiones con la pierna elevada, repeticiones con pierna elevada a diferentes objetivos en movimiento tales como pelotas de tenis de mesa, etc.). La premisa es que, al ejecutar posteriormente la destreza, sin la complejidad del contexto, su ejecución mejora.

Los microciclos de ajuste se ubican al iniciar los mesociclos. Al no introducir gestos 0 variantes nuevas, la persona tiene la posibilidad de realizar acciones que conoce y no -10 - 
está siempre expuesta a una alta exigencia en las sesiones, esto facilita el aprovechamiento y la mejor disposición en los microciclos de carga e impacto. Asimismo, los microciclos de activación son importantes para que la persona termine de integrar los estímulos recibidos durante el proceso de preparación, esté lista para el microciclo de competición y pueda desarrollar un óptimo desempeño.

$\mathrm{Si}$ los microciclos de carga son esenciales para el desarrollo de nuevas adaptaciones y cualidades, los microciclos de recuperación son igualmente importantes para propiciar un adecuado aprovechamiento de las sesiones, así como incentivar la adherencia hacia la práctica deportiva, ya que la ejecución de diversidad de actividades fomenta la motivación. En los practicantes de taekwondo muy orientados a la competición de combate, este tipo de microciclos son propicios para practicar "formas" y defensa personal, logrando el desarrollo integral que todo alumno debería tener.

\section{CONCLUSIÓN}

La adaptación del modelo ATR en el entorno educativo se considera una herramienta eficaz para apoyar el objetivo de formar técnica y tácticamente a las personas que se inician en la práctica deportiva y desarrollar en ellas el gusto por realizar actividad física. Aunque la experiencia del autor ha sido implementada este modelo en la disciplina del taekwondo, se considera que puede ser aplicado en cualquier otro arte marcial y, especialmente, en los deportes con importantes volúmenes de entrenamiento técnico.

En el entorno de las artes marciales, se debe desarrollar la capacidad de los competidores de razonar y tomar las decisiones oportunas al intervenir en un combate (Avelar-Rosa, Gomes, Figueiredo y López-Ros, 2015). Se comparte dicha posición y se considera que el modelo descrito favorece esta habilidad.

Al lograr desarrollar un clima motivacional favorable, el aprendizaje de las destrezas deportivas mejora (Meroño, Calderón y Hastie, 2016). Para lograr este ambiente idóneo, se debe evitar la monotonía y la repetición innecesaria de actividades, las lecciones deben ser entretenidas y favorecer la autonomía en la toma de decisiones (BaenaExtremera, Gómez-López, Granero-Gallegos y Martínez-Molina, 2016).

La presente manera de organizar las actividades dentro del proceso de enseñanza implica la constante variación en las dinámicas a realizar durante las lecciones; asimismo, la necesidad de establecer frecuentemente objetivos y contenidos de entrenamiento propicia la participación del deportista en la toma de decisiones; consecuentemente, favorece un adecuado contexto para desarrollar el proceso de enseñanza-aprendizaje.

Si bien la conceptualización del modelo teóricamente se presenta como un procedimiento adecuado para desarrollar en el ámbito del deporte con fines recreativos, de salud o formación base, la evidencia que apoya su efectividad se fundamenta en experiencias que no han sido sistemáticamente fundamentadas, por lo que es necesaria la corroboración de sus virtudes mediante evidencia experimental. 


\section{AGRADECIMIENTOS} modelo.

Agradezco a mis alumnos y alumnas, la experiencia compartida construyó este

\section{REFERENCIAS}

Avelar-Rosa, B., Gomes, M., Figueiredo, A. y López-Ros, V. (2015). Caracterización y desarrollo del "saber luchar": contenidos de un modelo integrado para la enseñanza de las artes marciales y de los deportes de combate. Revista de Artes Marciales Asiáticas, 10(1), 16-33. Doi: https://doi.org/10.18002/rama.v10i1.1501

Baena-Extremera, A., Gómez-López, M., Granero-Gallegos, A. y Martínez-Molina, M. (2016). Modelo de predicción de la satisfacción y diversión en Educación Física a partir de la autonomía y el clima motivacional. Universitas Psychologica, 15(2), 1525. Doi: 10.11144/Javeriana.upsy15-2.mpsd

Bompa, T., y Buzzichelli, C. (2015). Periodization Training for Sports (3 ${ }^{\text {ra }}$ ed.). Estados Unidos de América: Human Kinetics. Recuperado de: http://www.human kinetics.com/products/all-products/periodization-training-for-sports-3rd-edition-ebook

Campos, J. y Cervera, V. (2011). Teoría y planificacion del entrenamiento deportivo (4ta ed.). Barcelona, España: Editorial Paidotribo. Recuperado de: http://www.paidotribo.com/pdfs/537/537.i.pdf

Carazo-Vargas, P., González-Ravé, J. M., Moncada-Jiménez, J. y Newton, R. (2015). Periodization model for Costa Rican taekwondo athletes. Strength and Conditioning Journal, 37(3), 74-83. Doi: 10.1519/SSC.0000000000000139

Garber, C., Blissmer, B., Deschenes, M., Franklin, B., Lamonte, M., Lee, I., . . Swain, D. (2011). Quantity and quality of exercise for developing and maintaining cardiorespiratory, musculoskeletal, and neuromotor fitness in apparently healthy adults: guidance for prescribing exercise. Medicine y Science in Sports y Exercise, 43(7), 1334-1359. Doi: 10.1249/MSS.0b013e318213fefb.

García, J., Martínez, C., Meseguer, A. A., Ros, A., Rosa, A., y Rosa, M. (2018). Problemas de organización en gestión de equipos: Desalineación de objetivos en deporte base. Retos, 33, 148-151. Recuperado de: https://recyt.fecyt.es/index.php/ retos/article/view/55513/35805

González-Ravé, J. M., Pablos-Abella, C. y Navarro-Valdivieso, F. J. (2014). Entrenamiento Deportivo. Teorías y prácticas. Madrid, España: Panamericana. Recuperado de: https://www.medicapanamericana.com/Libros/Libro/5066/Entrenamiento-

Deportivo.html

Houston, M., Hoch, M., y Hoch, J. (2016). Health-Related Quality of Life in Athletes: A Systematic Review With Meta-Analysis. Journal of Athletic Training, 51(6), 442-453. Doi: https://doi.org/10.4085/1062-6050-51.7.03 
Issurin, V. (2010). New Horizons for the Methodology and Physiology of Training Periodization. Sports Medicine, 40(3), 186-206. Doi: 10.2165/11319770-00000000000000

Meroño, L., Calderón, A. y Hastie, P. (2016). Effect of Sport Education on the technical learning and motivational climate of junior high performance swimmers. Revista Internacional de Ciencias del Deporte, 12(44), 182-198. Doi: http://dx.doi.org/10.5232/ricyde2016.04407

Ranisavljevic, I. e llic, V. (2010). Periodization variants in strength training throughout microcycles and mesocycles. Proceedings, 2, 304-311. Recuperado de http://connection.ebscohost.com/c/articles/77466389/periodization-variants-strengthtraining-throughout-microcycles-mesocycles

Salazar, C. (2007). Recreación. San José, Costa Rica: Editorial Universidad de Costa Rica.

Theokas, C. (2009). Youth sport participation-a view of the issues: introduction to the special section. Developmental Psychology, 45(3), 303-306. Doi: 10.1037/a0015042 